\title{
Hemangioendotelioma Epitelioide Pulmonar Reporte de un caso
}

\author{
Victoria Monterroso-Azofeifa, ${ }^{1}$ Douglas Otero-Reyes, ${ }^{2}$ José Naranjo-Quirós,${ }^{3}$ Juan José Segura-Fonseca, ${ }^{4}$ Carlos \\ Salazar-Vargas ${ }^{5}$
}

\begin{abstract}
Resumen: En 1983 se reportaron 20 enfermos con un tumor pulmonar raro que fue entonces llamado IVBAT, por sus siglas en inglés (tumor intravascular bronquio-alveolar). Los pacientes se presentaron con numerosos nódulos pulmonares, de crecimiento lento, cuyos hallazgos histológicos variaban desde una apariencia benigna granulomatosa hasta una claramente maligna sarcomatosa.
\end{abstract}

Eventualmente se demostró que el tumor era de origen vascular y se le llamó hemangioendotelioma epiteliode (HEE). Poco después se describió en otras regiones anatómicas.

Reportamos aquí el primer caso costarricense de esta estirpe histológica y de presentación pulmonar.

Un hombre de 42 años de edad, nos fue referido en fase final con metástasis generalizadas, aunque en buenas condiciones generales. Se le practicó una biopsia pulmonar para definir la histología, pero falleció poco tiempo después sin responder a la terapia instituida. Dieciséis años antes se le habían descubierto nódulos pulmonares pequeños bilaterales y mediante una biopsia abierta se le hizo el diagnostico de HEE. Como el curso de su enfermedad fue considerado estable durante ese tiempo había sido seguido clínicamente.

Se comparó el material de la biopsia inicial con el de la reciente, encontrándose mucho mayor celularidad y atipia en la última. Se efectuaron análisis inmunohistoquímicos con un panel de anticuerpos, que permitió establecer la naturaleza vascular del tumor.

El HEE es un tumor muy raro, de crecimiento lento y de bajo potencial metastásico, a pesar de ser de origen vascular. Llama la atención el fenómeno de dependencia hormonal de este tumor, que se plantea en la literatura, y que sugiere otras avenidas terapéuticas.

Recibido: 27 de febrero de 2001

Aceptado: 17 de abril de 2001

En 1983 un grupo de patólogos reportó 20 pacientes de diferentes partes del mundo con un tipo particular de tumor pulmonar que llamaron IVBAT, por sus siglas en inglés, (tumor intravascular, bronquiolar y alveolar), que habían empezado a recolectar desde 1962.

La presentación más frecuente en este grupo fue de nódulos pulmonares bilaterales de crecimiento muy lento, que en

Abreviaturas: IVBAT, tumor intravascular bronquio-alveolar; HEE, hemangio-endotelioma epitelioide

Servicio de Anatomía Patológica, Hospital México.

Servicio de Oncología, Hospital México.

Servicio de Imágenes Médicas, Hospital México.

Servicio de Anatomía Patológica, Hospital San Juan de Dios.

Servicio de Cirugía de Tórax y Cardiovascular, Hospital México.

Correspondencia: Carlos Salazar Vargas. Apartado 1826-1250 Escazú. ocasiones eran encontrados fortuitamente, y que sugerían enfermedades granulomatosas a algunos observadores y metástasis a otros. La mayoría de los enfermos eran del sexo femenino y solo 2 eran mayores de 50 años.

Desde el punto de vista histológico, los pacientes les habían sido referidos con diagnósticos que variaban desde adenocarcinoma y sarcoma en lo maligno, hasta granulomas o infartos pulmonares en vías de organización, en lo benigno.

Corrin y cols. demostraron ultraestructuralmente en muestras de esos tejidos, los cuerpos de Weibel-Palade, ${ }^{2}$ los cuales son característicos de las células endoteliales. Posteriormente el desarrollo y la confirmación de marcadores endoteliales como la aglutinina de Ulex europeus, el CD31 y CD34 avalaron este origen. 
En 1982, Weiss y Enzinger acuñaron un mejor término para esta clase de tumor: Heman gioendotelioma Epitelioide (HEE). En esa serie los tumores estaban primariamente localizados en tejidos blandos, eran de un curso clínico intermedio entre hemangiomas y hemangiosarcomas y característicamente eran de baja malignidad. ${ }^{3}$

Luego se describió la presentación de HEE en ganglios linfáticos, cavidad oral, estómago, corazón y en órganos parenquimatosos como el bazo y el cerebro, ${ }^{3}$ y otros autores reportaron luego esta neoplasia en el hígado, ${ }^{4,5}$ el pene, ${ }^{6}$ la columna vertebral, ${ }^{7}$ el cerebro, ${ }^{8}$ el mediastino, ${ }^{9}$ la pleura ${ }^{10,11}$ y un caso de compromiso múltiple concomitante, sin poderse establecer el sitio original del primario. ${ }^{12}$ También se reportó el primer caso de un hemangioma epitelioide del corazón. ${ }^{13}$

Presentamos aquí, hasta donde hemos podido constatar, el primer paciente costarricense con un HEE, en este caso de localización pulmonar.

\section{Reporte del caso}

Un hombre de 42 años consultó con su médico por presentar discreta disnea de esfuerzo y dolor en la espalda y en las caderas, que atribuía a haber realizado labores poco habituales para él, relacionadas con la construcción de su casa. Se le practicó una tele-radiografía de tórax en octubre de 1998 y fue referido.

Dieciséis años antes, en un estudio radiológico de tórax de rutina, se le habían descubierto nódulos pulmonares, bien definidos, no calcificados, distribuidos bilateralmente, aunque con predominio del campo medio izquierdo. Su dimensión promedio era de $9 \mathrm{~mm}$. A raíz de ello fue sometido a una biopsia de la língula en el Hospital San Juan de Dios. Se hizo así el diagnóstico histológico de IVBAT, hoy conocido como HEE.

El paciente siguió en control anual clínico y radiológico, pero sin recibir tratamiento alguno, ya que la enfermedad aparentemente se mantuvo estable.

Había fumado durante 10 años, antes del diagnóstico y a partir de mayo de 1998, dejó de hacerlo. Ingería licor solo socialmente.

El examen físico en la presente ocasión fue negativo, excepto por discreta disminución de la ventilación, sobretodo en el campo pulmonar izquierdo, y por dolor a la presión sobre las caderas. Los exámenes de laboratorio estaban normales y el grupo sanguíneo era $\mathrm{B}+$. La nueva radiografía de tórax mostró múltiples nódulos de diverso tamaño en ambos campos pulmonares y la serie ósea evidenció lesiones metastásicas en cráneo, costillas, vértebras y huesos ilíacos.

La tomografía axial computarizada de tórax coincidió con las placas convencionales, excepción hecha de observarse un número mayor de lesiones y de la presencia de una masa hiliar izquierda (Figura 1). También se notaron numerosas áreas hipodensas hepáticas.
Debido a que el diagnóstico inicial era el de una neoplasia tan infrecuente y a la explosiva presentación actual de la enfermedad, después de tantos años de estabilidad, se decidió obtener una nueva biopsia, para ver si había habido un cambio histológico y reconfirmar el diagnóstico original. Se practicó entonces una pequeña toracotomía izquierda, encontrándose nódulos blancuzcos en ambos lóbulos y una masa hiliar, dependiente del lóbulo inferior, de los cuales se tomaron varias muestras. La evolución postoperatoria fue normal.

El material obtenido en 1982 aún se conservaba y fue comparado con el de 1998.

En los dos grupos de muestras el tumor exhibió el mismo patrón nodular general, compuesto por zonas centrales acelulares, hialinizadas, con microcalcificaciones ocasionales, rodeadas por grupos de células epitelioides con abundante citoplasma eosinofílico (Figuras 2, 3 y 4), en ambas muestras se encontraron algunas vacuolas intra-citoplasmaticas con eritrocitos en su interior. Se encontró invasión de las estructuras alveolares y de los vasos pequeños pero no de los grandes ni de los bronquiolos.

A pesar de las similitudes mantenidas a través del tiempo, encontramos también varias diferencias entre las biopsias iniciales y las actuales. En estas últimas, 1) los núcleos eran menos regulares, con una menor relación núcleo/citoplasma y con un nucleolo mucho más prominente, 2) había multinucleación ocasional, 3) se agregó la presencia de algunas mitosis típicas y atípicas, 4) se presentó una mayor densidad de la población celular, que además mostró zonas de un patrón alveolar, 5) había extensas zonas de necrosis, 6) se observó moderada infiltración por linfocitos y células plasmáticas y 7) había una leve reacción desmoplástica y algunos acúmulos de macrófagos espumosos.

En el material de biopsia reciente se efectuaron análisis inmunohistoquimicos (Figura 5). Las células tumorales expresaron vimentina, un marcador mesenquimal, y también factor VIII, un marcador endotelial, y no expresaron queratina, ni antígeno epitelial de membrana. Esto permitió confirmar la naturaleza endotelial del tumor y descartar entonces mesotelioma, adenocarcinoma, y melanoma, tumores que se incluían dentro del diagnóstico diferencial, que expresan queratina, pero no factor VIII.

Debido a la agresiva presentación de la enfermedad en esta etapa, se recomendó quimioterapia con "MAID" modificada (mesna, epirubicina, isofosfamida, y dacarbacina), planeada en ciclos cada 28 días.

El paciente recibió 2 ciclos de tratamiento, sin observarse respuesta, sino más bien progresión clínica de la enfermedad. Posteriormente, hizo metástasis submentonianas y a cuero cabelludo, y un derrame pleural izquierdo. Falleció en febrero de 1999 , en su casa y no se practicó autopsia. 


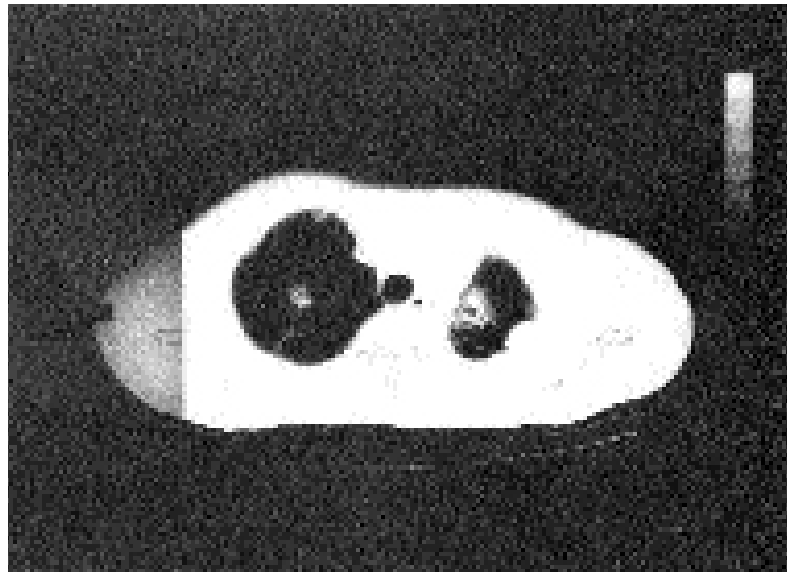

Figura 1. El estudio muestra una densidad focal (nódulo) en lóbulo superior derecho de 19x12 $\mathrm{mm}$ de bordes irregulares y de componente mixto. El campo izquierdo muestra un contorno pleural ondulado representativo de hidrotórax loculado.

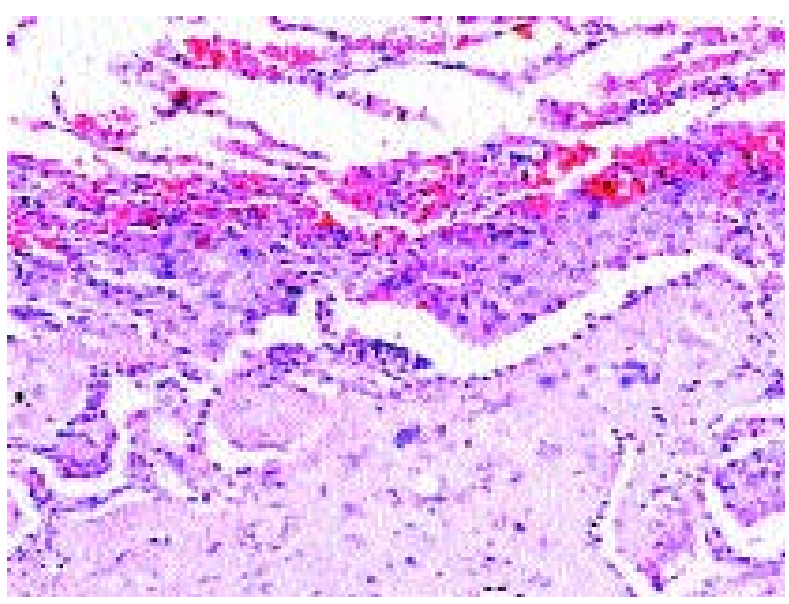

Figura 2. Pulmón, H\&E, 40x. Se observa uno de los nódulos pulmonares de la biopsia inicial, efectuada 16 años antes, que muestra poca celularidad y presencia de material eosinofílico amorfo. Hay parénquima pulmonar comprimido en la periferia.

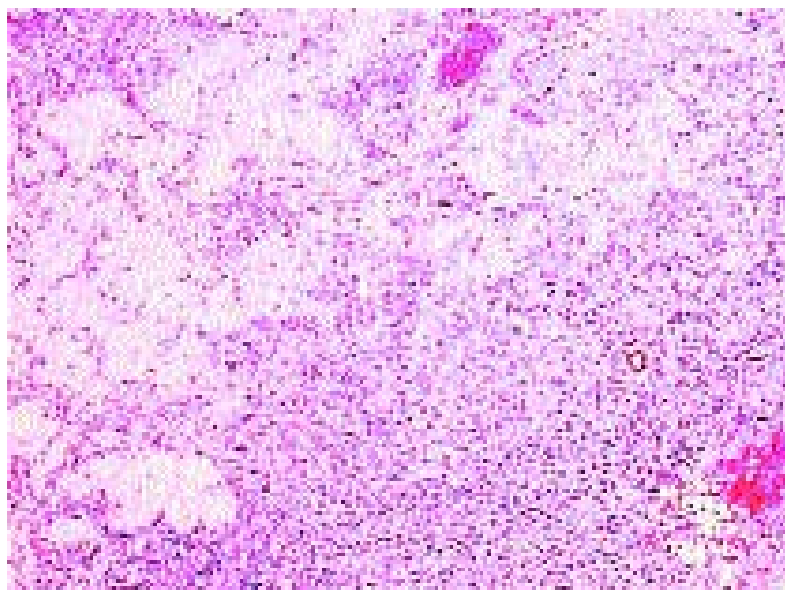

Figura 3: Pulmón, H\&E, 40x. Uno de los nódulos de la biopsia reciente, con mucho mayor celularidad, aunque siempre con áreas acelulares.

\section{Discusión}

Aspectos clínicos: El HEE es un tumor raro de origen endotelial, que puede desarrollarse en cualquier tejido pero es más común en el hígado y en el pulmón, su comportamiento depende del órgano primario de origen, pero en general su curso es lento. ${ }^{3}$

Cuando se origina en los tejidos blandos, ocurre en cualquier parte del cuerpo, siendo entonces más fácil de diagnosticar porque aparece como una tumoración dura y frecuentemente dolorosa, de crecimiento lento, a veces de años de evolución. En estos casos la excisión y radioterapia es capaz de controlar el tumor. De 24 casos seguidos por 36 meses, solamente 4 pacientes murieron de enfermedad tumoral. ${ }^{14}$

Se ha descrito que si el sitio primario es de tejidos blandos, la mortalidad a los 4 años de seguimiento es de $13 \%, 35 \%$ si es

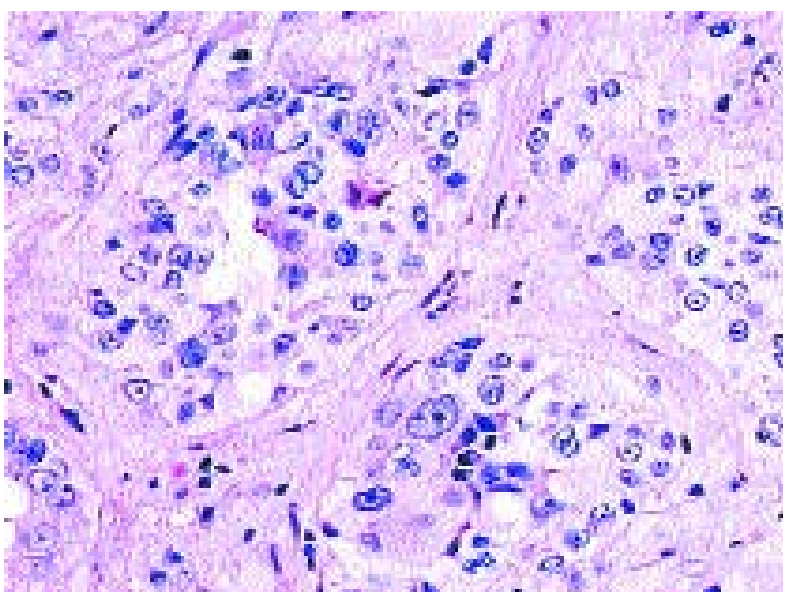

Figura 4: Pulmón, H\&E, 400x. Mayor aumento de las áreas celulares de la Figura 3 donde se aprecia marcada atipia de las células que revisten los espacios vasculares.

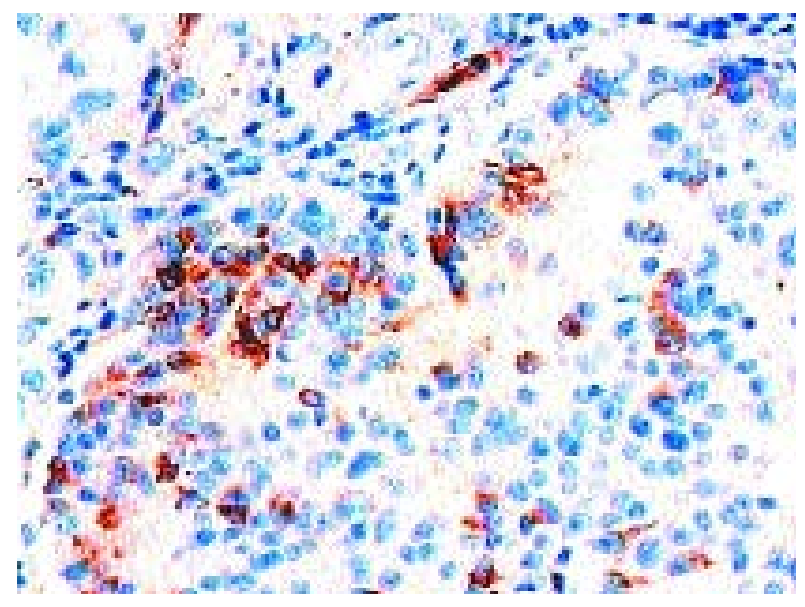

Figura 5. Pulmón, inmunoperoxidasa con anti-factor VIII contrastada con hematoxilina, 100x. Algunas de las células tumorales que revisten los espacios expresan factor VIII en la membrana celular y el citoplasma (coloración pardo rojiza). 
del hígado y $65 \%$ si el primario es en el pulmón. ${ }^{11}$ Una vez que el tumor permea los vasos, lentamente destruye el órgano original, muriendo el paciente de insuficiencia hepática, ${ }^{5} \mathrm{o}$ respiratoria, ${ }^{1}$ según sea el caso.

En el caso del HEE de origen óseo, aunque tiende a ser multifocal, cursa lentamente, y tiene bajo potencial metastático. ${ }^{12}$

El otro aspecto interesante de esta neoplasia, es su restringida capacidad de metastatizar, ya que a pesar de ser un tumor vascular por definición, y por ende con amplio acceso al torrente sanguíneo, no todos lo hacen. Esto sucede solamente en el $20 \%$ de los casos de tejidos blandos, el $15 \%$ de los originarios del pulmón y el $25 \%$ de los hepáticos. Los sitios preferidos de las metástasis son los ganglios linfáticos, el hígado y los pulmones. ${ }^{15}$

Como el curso es lento y frecuentemente silencioso desde el punto de vista clínico, en ocasiones al presentarse el paciente ya con enfermedad diseminada, es difícil determinar el origen del primario y entonces se cataloga como un primario multicéntrico simultáneo. ${ }^{12}$

Tanto el HEE hepático como el pulmonar muestran una fuerte preferencia por el sexo femenino. ${ }^{12}$ Este hecho aunado a observaciones de la aparición de esta neoplasia en mujeres que usaron contraceptivos orales, sugiere una posible influencia hormonal en el desarrollo de los mismos. ${ }^{16}$ De hecho Ohori y cols. reportaron receptores estrogénicos en el tejido de una de 5 pacientes con este tumor.

\section{Aspectos radiológicos}

Los hallazgos radiológicos pulmonares de nuestro paciente coinciden con los descritos en los pacientes de la serie original de Dail y cols. Básicamente, se encuentran numerosos nódulos, no calcificados, menores de $20 \mathrm{~mm}$ de diámetro y la ausencia de adenopatías mediastinales. ${ }^{1}$

Ciertamente de no haber existido el conocimiento previo de la histología, en este paciente, esta rara entidad no se habría propuesto, sino más bien el diagnostico diferencial radiológico sería entre nódulos granulomatosos y actividad metastásica de otro origen primario. El hecho de haber descubierto invasión del hígado, nos permite sugerir el tamizaje abdominal más tempranamente.

Recientemente otros autores han descrito una presentación radiológica diferente, que puede confundirse con un proceso inflamatorio o intersticial, y que posiblemente represente una variedad de tumor más agresivo. ${ }^{18}$

\section{Aspectos terapéuticos}

Si el tumor está bien localizado y es factible su extirpación completa, esta debe de realizarse.

Recientemente se ha reportado el trasplante hepático como tratamiento para el HEE de dicho órgano. ${ }^{19}$
Debido a que es un tumor de tejidos blandos, de bajo grado de malignidad, pero con un curso intermedio entre hemangioma $\mathrm{y}$ angiosarcoma, algunos investigadores han utilizado quimioterapia en su tratamiento. El esquema estándar recomendado para sarcomas de partes blandas ha sido el "MAID", que consiste en mesna, adriamicina, isofosfamida, y dacarbacina, ${ }^{18}$ siendo el primer medicamento un reno protector, y el último actualmente ha sido eliminado del régimen, porque no aporta beneficio y por otro lado aumenta la toxicidad. Debido a la rápida y agresiva presentación de nuestro enfermo se optó por una quimioterapia intensa para tratar de obtener una respuesta objetiva, se utilizó MAI sustituyendo doxorubicina por epirubicina, sin embargo el paciente no respondió. Se reportan casos de respuesta completa de tumores en diferentes regiones a la quimioterapia. ${ }^{12,21}$

\section{Aspectos anatomopatológicos}

Los pacientes referidos a los autores que originalmente describieron esta entidad presentaban nódulos, característicamente múltiples, menores de $2 \mathrm{cms}$ de diámetro, sin extensión microscópica vascular o bronquial. Histológicamente estos tenían zonas centrales acelulares, hialinizadas o mixoides, rodeadas por cantidad variable de células de aspecto epitelioide, con citoplasma claro o granular y núcleos redondos u ovalados, sin atipias, con nucleolos ausentes o incospicuos, dispuestas en nidos y cordones. Las mitosis eran escasas. ${ }^{1}$

Dos hallazgos histológicos sugirieron a estos autores el posible origen vascular de este tumor: la presencia de vacuolas citoplasmáticas, algunas de ellas con eritrocitos en su interior; y un grado variable de permeación vascular.

En el mismo estudio demostraron, por análisis inmunohistoquímico, que las células tumorales y las vacuolas intracitoplásmicas expresaban el antígeno relacionado con el factor VIII.

Un criterio importante para el diagnóstico es la evidencia inmuno histoquímica de diferenciación endotelial.y aunque el marcador más específico y sensible es el CD31 algunas lesiones vasculares son negativas y deberán estudiarse con otros marcadores como el factor VIII o el Ulex europeus.

En este caso, en el que fue posible seguir la evolución histopatológica a través de 16 años, se notaron varias características que indican una progresión a un estado tumoral más agresivo, como son: mayor atípica celular, presencia de mitosis típicas y atípicas, no observadas en el tumor inicial y zonas de necrosis indicativas de un crecimiento acelerado por encima de su capacidad de irrigación. Además se evidenciaron dos tipos de reacción del organismo contra el tumor: un infiltrado linfo/plasmocitario denso zonal y una leve respuesta desmoplásica.

En conclusión el HEE es un tumor maligno de origen vascular, de tejidos blandos o de cualquier órgano, es muy poco frecuente, se presenta más en mujeres, tiene una influencia hormonal, y es de lenta evolución. 


\section{Abstract}

In 1983 an uncommon type of lung tumor was reported in 20 patients, it was then named IVBAT (intravascular, bronchioalveolar tumor). The patients presented with multiple slow growing pulmonary nodules, that ranged microscopically from (apparently) benign looking granulomas to clearly developed sarcomas.

Eventually the tumor was found to be of vascular origin, and was called epithelioid hemangio endothelioma (EHE), later on it was also reported in other anatomical regions.

We describe here, as far as we could find, the first costarican case of this particular neoplasia.

A 42 year old male was referred to us with disseminated metastatic disease. He had been seen 16 years prior with asymptomatic, bilateral, small lung nodules. At that time he underwent a lung biopsy and the diagnosis of epitheliod hemangioendothelioma was made. He had remained stable, on no therapy, until his current and final presentation. We elected to biopsy his lung again to verify the histology and to see weather the tumor had undergone differentiation. He did not respond to chemotherapy and died shortly thereafter.

The original lung biopsy material was compared to the recent one, finding much more cellularity and atypiae in the latter one. Immuno-histochemistry studies were carried out on the tissue samples and the endothelial origin of the tumor was demonstrated.

EHE is an unusual type of neoplasia, it grows very slowly and has low metastatic potential in spite of its vascular origin, interestingly some authors have shown a hormonal dependency and this needs to be explored to see if therapeutic applications can be developed.

\section{Referencias}

1. Dail DH, Liebow AA, Gmelich JT, Friedman PJ, Miyai K, Myer W, et al. Intravascular, bronchiolar, and alveolar tumor of the lung (IVBAT): an analysis of 20 cases of a peculiar sclerosing endothelial tumor. Cancer 1983 ; 51 : 452-464.

2. Corrin B, Manners B, Millard M, Weaver L. Histogenesis of the socalled "intravascular bronchioalveolar tumor". J Pathol 1979; 128: 163-167.

3. Weiss SW., Enzinger FM. Epithelioid hemangioendothelioma: a vascular tumor often mistaken for a carcinoma. Cancer 1982; 50: 970-981.

4. Ishak Kg, Sesterhenn Ia, Goodman ZD, Rabin L, Stromeyer FW. Epithelioid hemangioendothelioma of the liver: a clinico-pathologic and follow up study of 32 cases. Hum Pathol 1984; 15: 839-842.

5. Dietze O, Davies SE, Williams R, Portmann B. Malignant epithelioid hemangioendothelioma of the liver: a clinicopathological and histochemical study of 12 cases. Histopathology 1988; 15:225-237.

6. Haidar A, Batman P. Epithelioid hemangioendothelioma of the penile shaft. Brit J Urol 1995; 5 (6): 796-797.
7. Ellis Ts, Schwartz A, Starr JK, Riedel CJ. Epithelioid hemangioendothelioma of the vertebral column: case report and review of the literature. Neurosurgery 1996; 38 (2): 402-407.

8. Nora FE, Scheithauer BW. Primary epithelioid hemangioendothelioma of the brain. Am J Surg Pathol 1996; 20 (6): 707-714.

9. Begbie SD, Bell DR, Nevell DF. Mediastinal epithelioid hemangioendothelioma in a patient with type IV Ehlers-Danlos syndrome: a case report and review of the literature. Am J Clin Oncol 1997; 20 (4): 412415.

10. Pinet C, Magnan A, Garbe L, Payan MJ, Vervloet D. Aggressive form of pleural epithelioid hemangioendothelioma: complete response after chemotherapy. Eur Respir J 1999; 14 (1):237-238.

11. Crotty EJ, McAdams HP, Erasmus JJ, Sporn TA, Roggli VL. Epithelioid hemangioendothelioma of the pleura. AJR 2000; 175: 1545-1549

12. Bollinger BK, Laskin WB, Knight CB. Epitheliod hemangioendothelioma with multiple site involvement. Cancer 1994; 73: 610-615.

13. de Nictolis M, Brancorsini D, Goteri G, Prat J. Epithelioid hemangioma of the heart. Virchows Arch 1996; 428 (2): 119-123.

14. Mentzel T, Beham A, Calonje E, Katenkamp D, Fletcher CDM. Epitheliod hemangioendothelioma of skin and soft tissues: clinicopathologic and immunohistochemical study of 30 cases. Am J Surg Pathol 1997; 21 (4): 363-374.

15. Weiss SW, Ishak KG, Dalil GH, Sweet DE, Enzinger FM. Epithelioid hemangioendothelioma and related lesions. Semin Diagn Pathol 1986; 3: 259-287.

16. Dean PJ, Haggitt RC, O_Hara CJ. Malignant epithelioid hemangioendothelioma of the liver in young women: relationship to oral contraceptive use. Am J Surg Pathol 1985; 9: 695-704.

17. Ohori NP, Yousem SA, Sonmez-Alpan E, Colby TV. Estrogen and progesterone receptors in lymphangioleiomyomatosis, epitheliod hemangioendotheliloma and sclerosis hemangioma of the lung.Am J Clin Pathol 1991; 96: 529-535.

18. Mukundan G, Urban BA, Askin FB, Fishman EK. Pulmonary epithelioid hemangioendothelioma: atypical radiological findings of a rare tumor with pathologic correlation. J Computer Assisted Tom 2000; 24 (5): 719-720.

19. Hung CF, Jeng LB, Lee WC, Lin DY, Tan PP, Chen MF. Liver transplantation for epitheliod hemangioendothelioma. Transplant Proc 1998; 30 (7): 3307-3309.

20. Elias A, Ryan L, Sulkes A, Collins J, Aisner J, Antman KH. Response to mesna, doxorubicin, ifosfamide, and dacarbazine in 108 patients with metastatic or unresectable sarcoma and no prior chemotherapy. $\mathbf{J}$ Clin Oncol 1989; 7 (9): 1208-1216.

21. Idilman R, Dokmeci A, Beyler AR, Bastemir M, Ormeci N, Aras N, Ekinci C, Uzunalimoglu O, De Maria N, Van Thiel DH. Succesful medical treatment of an epitheliod hemangioendothelioma of the liver. Oncology 1997; 54 (2): 171-175. 